Supporting information

\title{
A novel salt-rejecting linen fabric-based solar evaporator for stable and efficient water desalination under highly saline water
}

Liang Song, Peng Mu*, Le Geng, Qingtao Wang, Jian Li*

Key Laboratory of Eco-functional Polymer Materials of the Ministry of Education,

College of Chemistry and Chemical Engineering, 967 Anning East Road,

Northwest Normal University, Lanzhou 730070, P. R. China.

*E-mail: pengmu2019@nwnu.edu.cn (P. Mu); Tel: +869317971533.

*E-mail: jianli83@126.com (J. Li); Tel: +869317971533.

Contents of the Supporting Information

Total number of pages: 16

Total number of figures: 16

Total number of tables: 4 


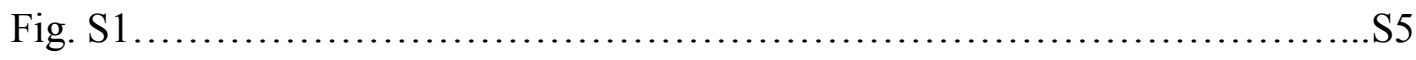

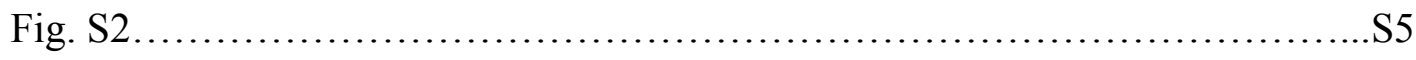

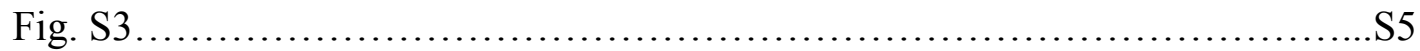

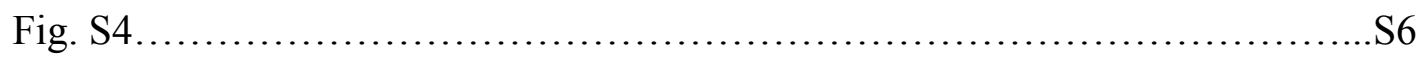

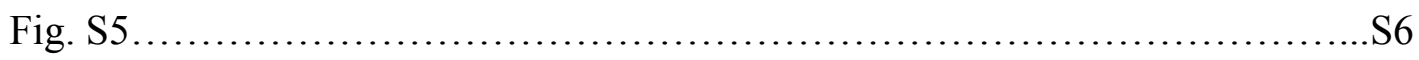

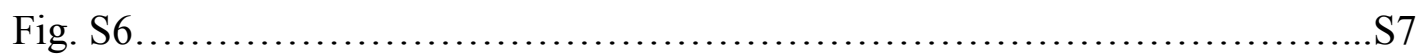

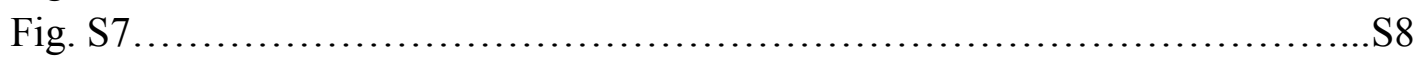

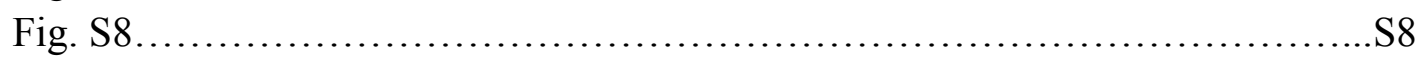

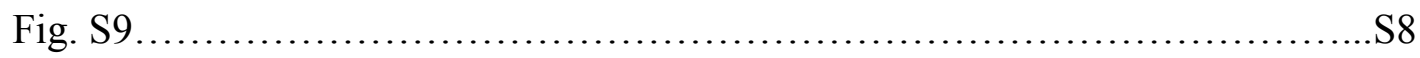

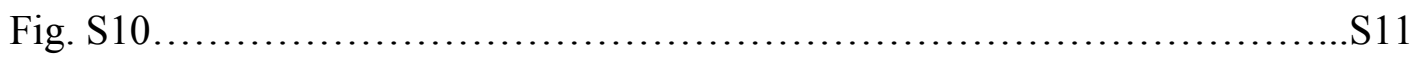

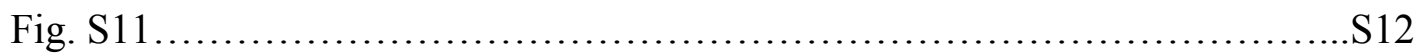

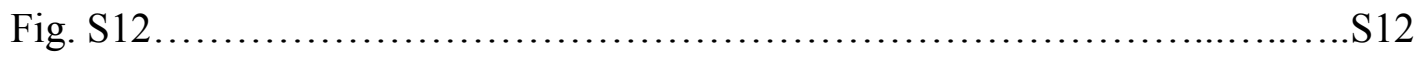

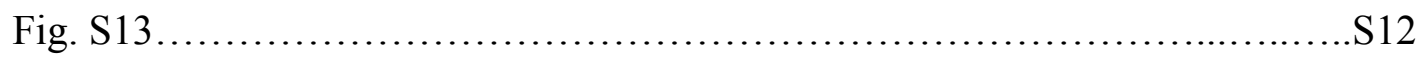

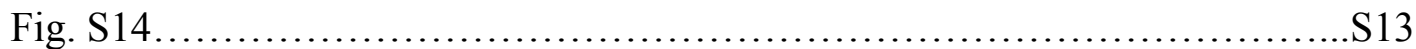

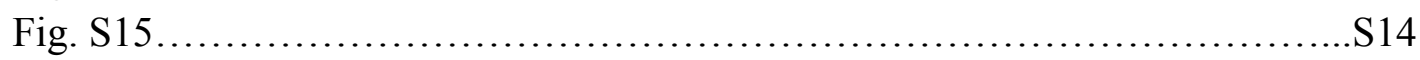

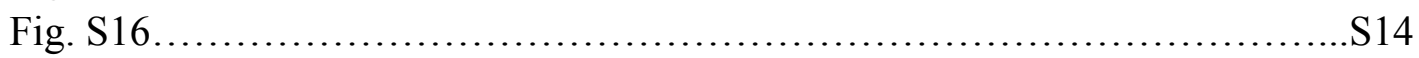

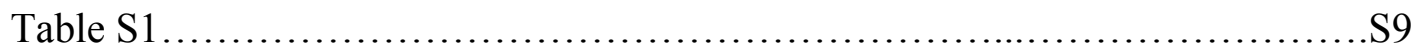

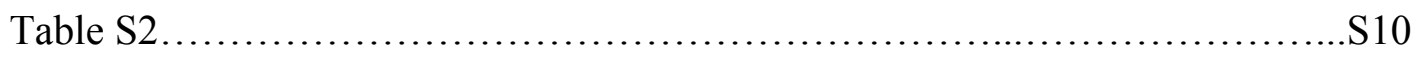

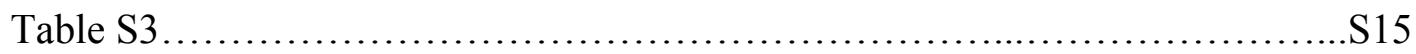

Table S4 ............................................................... 15 


\section{Solar steam generation test}

The solar steam generation experiments was conducted at a lab-made, online, real-time measurement system which is consists of a light simulation system and a detection system. The light simulation system including a solar light simulator (Xenon arc lamp, CEL-S500, Ceaulight) with a solar filter (AM 1.5, Ceaulight) and a light intensity was measured by a full spectrum optical power meter (CEL-NP2000-2, Beijing Education Au-light Co., Ltd.) The detection system including a test chamber is an open cuboid (30 $\mathrm{mm} \times 30 \mathrm{~mm} \times 80 \mathrm{~mm})$, an analytical balance (FA 2004 ), a computer to record the time-dependent mass change of water due to the stream generation and an infrared camera (Testo 869, Germany). During all tests, the room temperature was maintained at $19-22{ }^{\circ} \mathrm{C}$ and the humidity was ranged from 30 and $35 \%$.

\section{Calculation of the energy conversion efficiency}

$$
\mathrm{y}=\mathrm{mh}_{\mathrm{Lv}} / \mathrm{C}_{\mathrm{opt}} \mathrm{q}_{\mathrm{i}}
$$

where $\mathrm{m}$ is the mass flux of steam(the rate of water evaporation under the dark environment is subtracted), $\mathrm{C}_{\mathrm{opt}}$ is the optical concentration, $\mathrm{q}_{\mathrm{i}}$ is the nominal direct solar irradiation $1 \mathrm{~kW} \mathrm{~m}^{-2}, \mathrm{~h}_{\mathrm{Lv}}$ denotes total enthalpy of liquid-vapor phase change (including sensible heat and phase-change enthalpy), can be calculated as

$$
\mathrm{h}_{\mathrm{Lv}}=\lambda+\mathrm{C} \Delta \mathrm{T}
$$

where $\lambda$ is latent heat of phase change (The latent heat varies from $2430 \mathrm{~kJ} / \mathrm{kg}$ at $30{ }^{\circ} \mathrm{C}$ to $2265 \mathrm{~kJ} / \mathrm{kg}$ at $\left.100{ }^{\circ} \mathrm{C}\right), \mathrm{C}$ is specific heat capacity of water $(4.2 \mathrm{~kJ}$ $\mathrm{kg}^{-1} \cdot \mathrm{K}^{-1}$ ), and $\Delta \mathrm{T}$ denotes the temperature increase of the water.

\section{Calculation of the light absorption ability}

The light absorptivity of the membrane can be calculated by using the equation:

$$
A=1-R-T
$$

where $A$ is the absorbance, $R$ is the reflectance, and $T$ is the transmittance.

\section{Quantitatively heat loss analysis for the LFSTM evaporator under 1 sun irradiance.}

The heat loss of the evaporator contains (1) radiation, (2) convection and (3) conduction. The calculation details of heat loss are shown below.

\section{Radiation:}

The radiation loss was analyzed by Stefan-Boltzmann equation. The room temperature is about $21^{\circ} \mathrm{C}$. Supposing the evaporator has a maximum emissivity of 1 .

$$
\Phi=\varepsilon A \sigma\left(\mathrm{T}_{1}{ }^{4}-\mathrm{T}_{2}{ }^{4}\right)
$$

Where $\Phi$ is heat flux ( $\left.\mathrm{W} \mathrm{m}^{-2}\right), \varepsilon$ denotes emissivity, $\mathrm{A}$ is the surface area of the absorber facing the sun $\left(0.0004 \mathrm{~m}^{2}\right), \sigma$ is the Stefan-Boltzmann constant $\left(5.67 \times 10^{-8}\right.$ $\mathrm{W} \mathrm{m}^{-2} \mathrm{~K}^{-4}, \mathrm{~T}_{1}(316.15 \mathrm{~K})$ is the surface temperature of the evaporator at steady state under 1 sun illumination, and $\mathrm{T}_{2}(307.15 \mathrm{~K})$ is the ambient temperature upward the 
material under 1 sun illumination. According to the equation (S4), the radiation heat loss was calculated to be about $\sim 8 \%$ of all received solar energy.

\section{Convection:}

The heat loss of convection is caused by the air flowing and was analyzed by the following Newton' law of cooling.

$$
\mathrm{Q}=\mathrm{hA} \Delta \mathrm{T}
$$

Where $\mathrm{Q}$ is the heat energy, $\mathrm{h}$ is the natural convection heat transfer coefficient $(\sim$ $\left.5-10 \mathrm{~W} \mathrm{~m}^{-2} \mathrm{~K}^{-1}\right)$. A is the surface area of the absorber facing the sun $\left(0.0004 \mathrm{~m}^{2}\right)$. $\Delta T$ is the different value between the surface temperature and the ambient temperature upward the material under 1 sun illumination $(\Delta T=9 \mathrm{~K})$. Based on the above equation (S5), the heat loss of convection of the evaporator is $\sim 6 \%$ of all received solar energy.

\section{Conduction:}

As to the conduction to the underlying water, we estimate it to be $\sim 5 \%$ according to a very similar solar-thermal evaporation system where the polystyrene foam $(0.03$ $0.04 \mathrm{~W} \mathrm{~m}^{-1} \mathrm{~K}^{-1}$ ) with $1 \mathrm{~cm}$ thickness has the conductive heat loss is $\sim 5 \%$. (Proc Natl Acad Sci U S A 2016, 113 (49), 13953-13958.)

It should be noted that there is a large discrepancy between the efficiencies calculated by (100\%-calculated heat loss) $(\sim 19 \%)$ and the energy efficiency $(\sim 96 \%$ at 1 sun) calculated by the evaporation rate according to the formula commonly used in this field $\left(\mathrm{y}=\mathrm{m} h_{L v} / \mathrm{C}_{\mathrm{opt}} \mathrm{q}_{\mathrm{i}}\right)$. When we checked the literature and calculated the available data, we found that this large discrepancy was a common phenomenon.( Chem Sci 2018, 9 (3), 623-628; Nano Energy 2019, 56, 708-715.) On one hand, this discrepancy may come from the errors of the empirical formulas or coefficients used to calculate the heat losses and experimental errors. On the other hand, it should be pointed out that the commonly used formula to calculate evaporation efficiency depends on evaporation rate, which can be greatly affected by experimental conditions such as humidity, room temperature and steam diffusion rate. When comparing the efficiency of different systems, it is better to check the heat loss at the same time. For the calculation of heat loss, only a rough range can be estimated as much as possible and cannot be accurately estimated. 


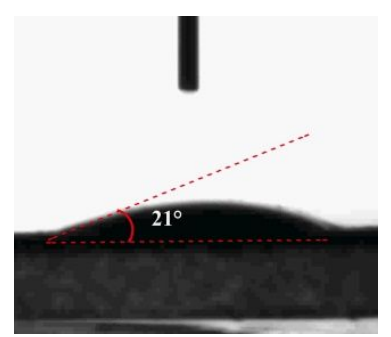

Figure S1. Contact angle images of candle soot. The contact angle of $21^{\circ}$ indicates the superhydrophility of LFS, which is conducive to sufficient water supply.

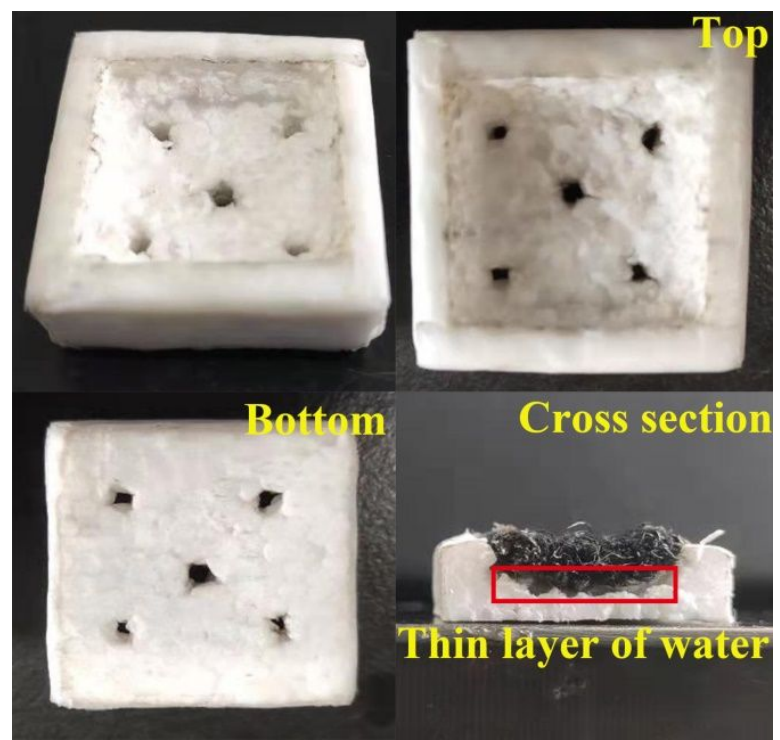

Figure S2. Optical images of the top, bottom, and cross-sectional of the as-prepared LFSTM. The side-length of the thermal insulator is $2 \mathrm{~cm}$ and the surface ratio of holes over the bottom was designed to be 1:25 for heat localization, as well as for sufficient water supply and removal salt. Clearly, the water thin layer is located between solar evaporator and thermal insulator.

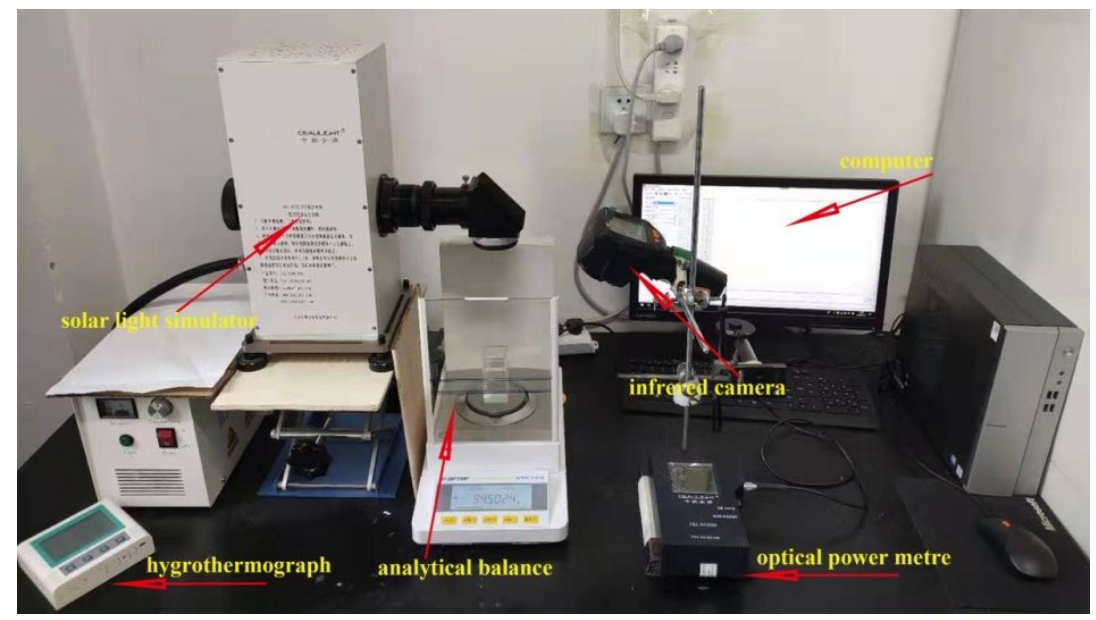

Figure S3. Schematic diagram of the equipment for solar steam generation performance testing 


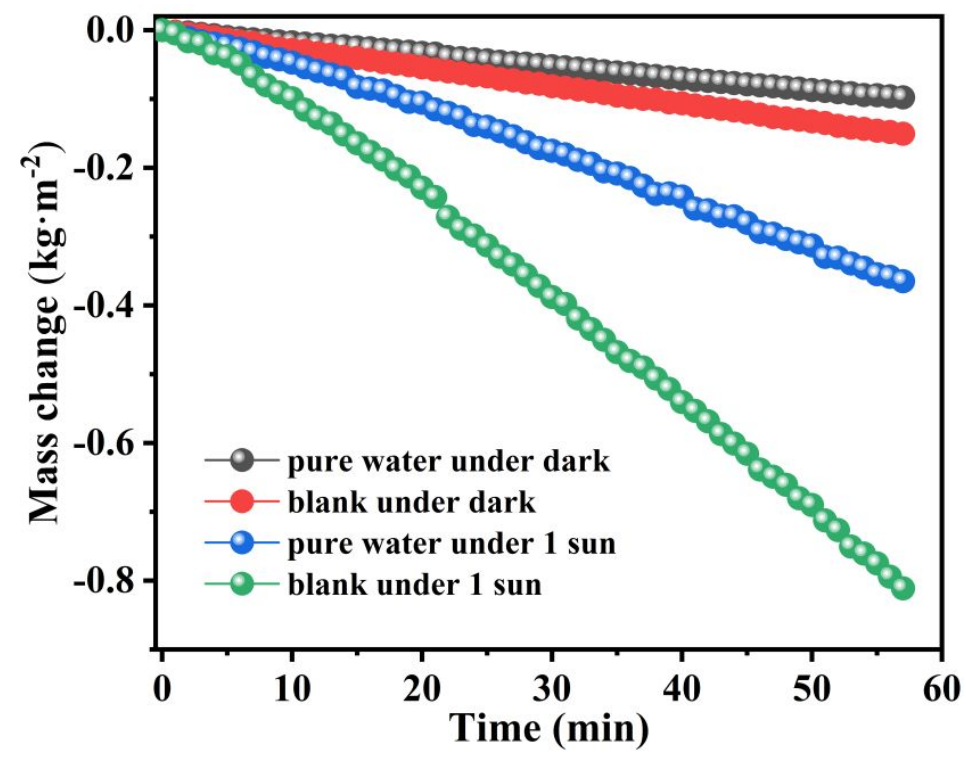

Figure S4. Time-dependent mass change of the pure water and blank under 1 sun irradiation or dark condition.
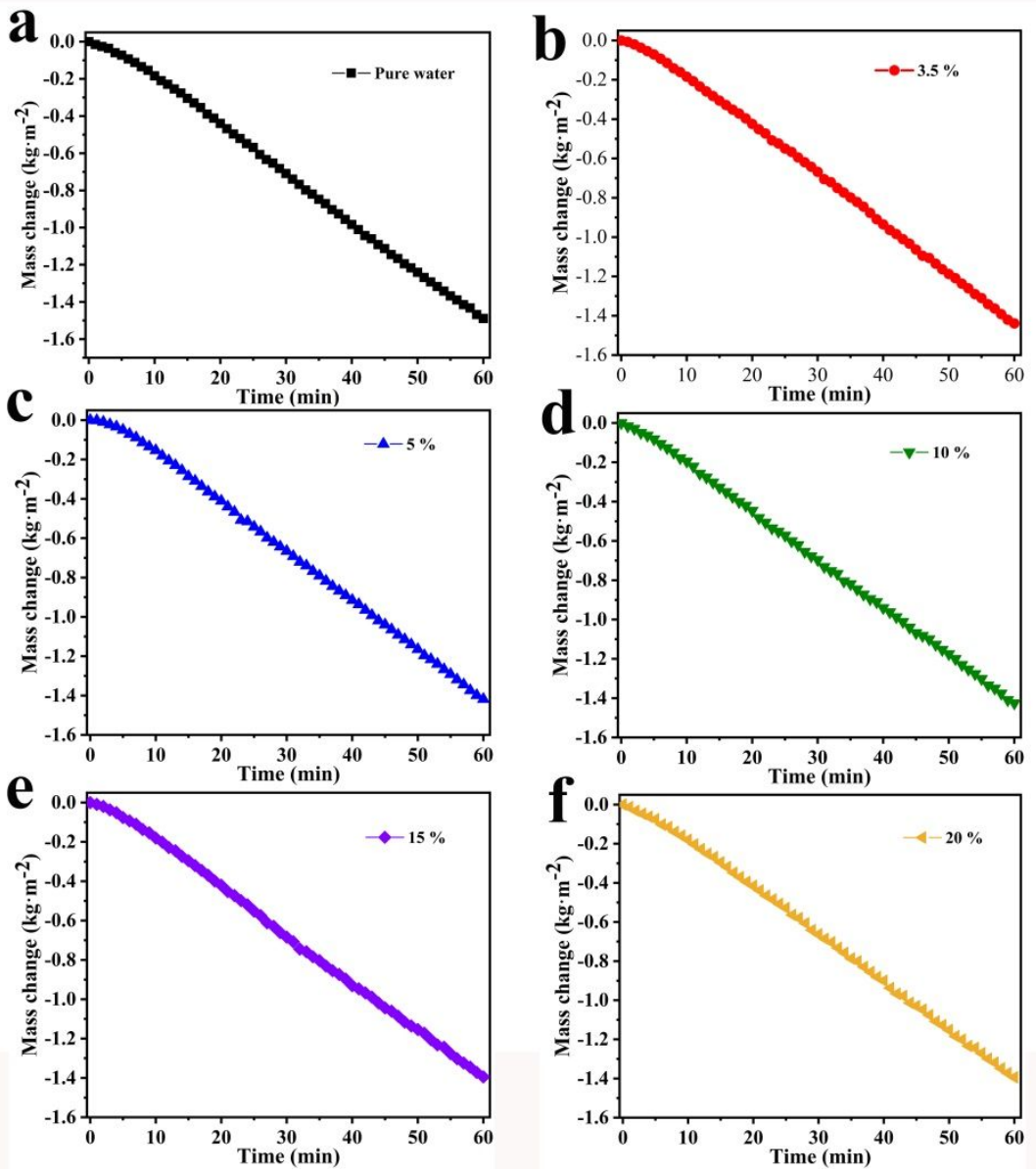

Figure S5. Time-dependent mass change for pure water (a), $3.5 \%$ (b), $5 \%$ (c), 10\% (d), $15 \%$ (e), and 20\% (f) saline water under 1 sun irradiation. 
The way to increase energy conversion efficiency is to improve thermal management and water supply. Therefore, the design and fabricate of solar evaporator for balancing enhanced thermal management and sufficient water supply still require further exploration. In our work, the thermal management and water supply is determined by the number of holes and thickness of thermal insulation device, respectively.

\section{Optimization of thickness:}

In this experiment, we estimated the solar evaporation rate and salt-rejecting performance of LFSTM (with different thickness of thermal management device $(1.5 \mathrm{~cm}, 1 \mathrm{~cm}$ and $0.5 \mathrm{~cm})$ ) for $20 \%$ saline water under 1 sun illumination.(i.e. the thermal management device with a thickness of $1.5 \mathrm{~cm}$ is donated as LFSTM $_{1.5}$ ). As shown in Fig. S6, for clarity, the water was stained with methylene blue and the yellow line indicated the water level. The water level is lower than the top level of LFSTM $_{1.5}$, which would lead to insufficient water supply and salt deposition. The water level and the top level of LFSTM $_{1.0}$ coincide, which would lead to sufficient water supply. The water level is higher than the top level of $\operatorname{LFSTM}_{0.5}$, which would lead to sufficient water supply and form a layer of water on the evaporation surface to increase reflection of light (Angew. Chem. Int. Ed. 2019.). As shown in Fig. S7, only the balance of buoyancy and gravity can ensure proper water supply. These inferences will be further proved by the evaporation rate of water and the phenomenon of salt deposition. As shown in Fig.S8, after 1 hour irradiation, we can clearly see that the salt deposited on the surface of LFSTM $_{1.5}$, while no salt deposited on the surface of $\operatorname{LFSTM}_{1.0}$ and $\operatorname{LFSTM}_{0.5}$. This difference is mainly due to insufficient water supply in $\operatorname{LFSTM}_{1.5}$. From the perspective of water evaporation rate, as shown in Fig. S9, the evaporation rate was calculated to be $1.300 \mathrm{~kg} \cdot \mathrm{m}^{-2} \cdot \mathrm{h}^{-1}, 1.453 \mathrm{~kg} \cdot \mathrm{m}^{-2} \cdot \mathrm{h}^{-1}$ and $1.386 \mathrm{~kg} \cdot \mathrm{m}^{-2} \cdot \mathrm{h}^{-1}$ for LFSTM $_{1.5}$, LFSTM $_{1.0}$ and $\mathrm{LFSTM}_{0.5}$ as a solar receiver for solar steam generation under 1 sun irradiation , respectively. The evaporation rate of $\mathrm{LFSTM}_{1.5}$ is lower than that of $\mathrm{LFSTM}_{1.0}$. This may be due to the precipitated salts can block water supply channels and reduce light absorption. The evaporation rate of $\mathrm{LFSTM}_{0.5}$ is lower than that of LFSTM $_{1.0}$, which can be attributed to the sufficient water supply and the forming of the thin layer of water on the evaporation surface to increase reflection of light. In summary, we choose LFSTM $_{1.0}$ as solar evaporator for high evaporation rate as well as salt-rejecting.

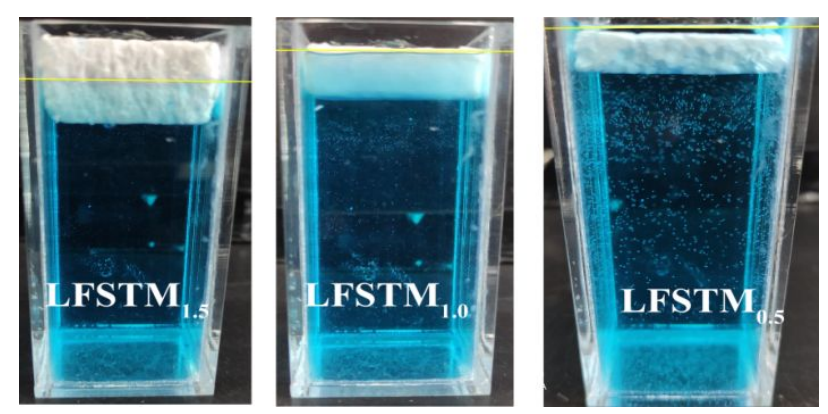

Figure S6 Digital photographs of the $\operatorname{LFSTM}_{(1.5-0.5)}$ with different thickness floating on water. Water stained with methylene blue. 


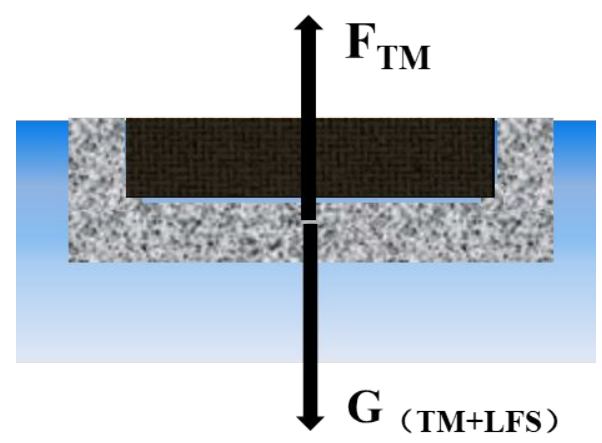

$$
\mathbf{F}_{\mathrm{TM}}=\mathbf{G}_{(\mathbf{T M}+\mathbf{L F S})}
$$

Figure S7 Force analysis of the LFSTM device. FTM and $\mathrm{G}_{(\mathrm{TM}+\mathrm{LFS})}$ is the buoyancy and gravity of the LFSTM device, respectively.

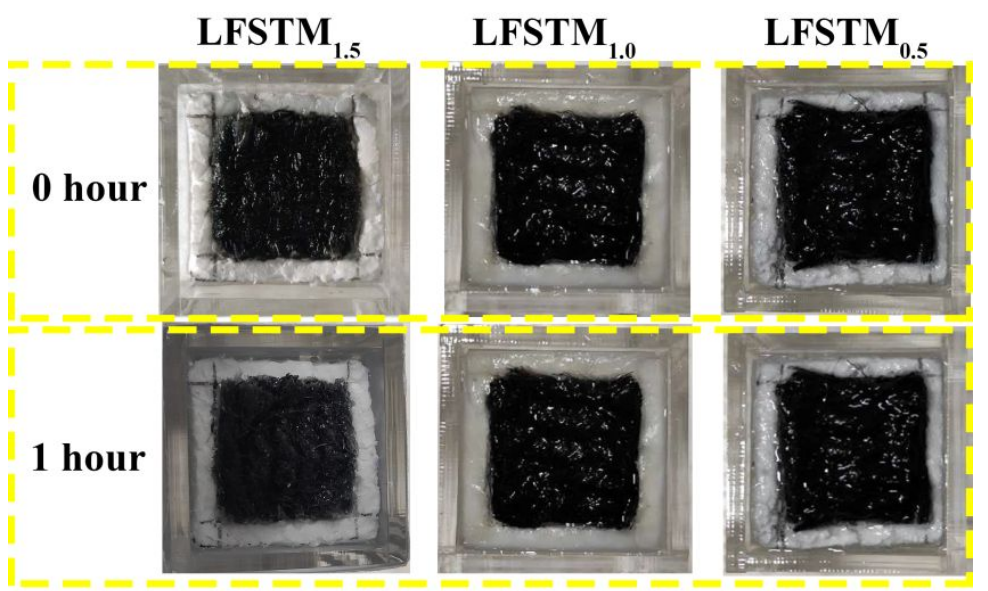

Figure S8 Digital photographs of the LFSTM $_{1.5}$, LFSTM $_{1.0}$ and LFSTM $_{0.5}$ under 1 sun irradiation before and after 1 hour evaporation.

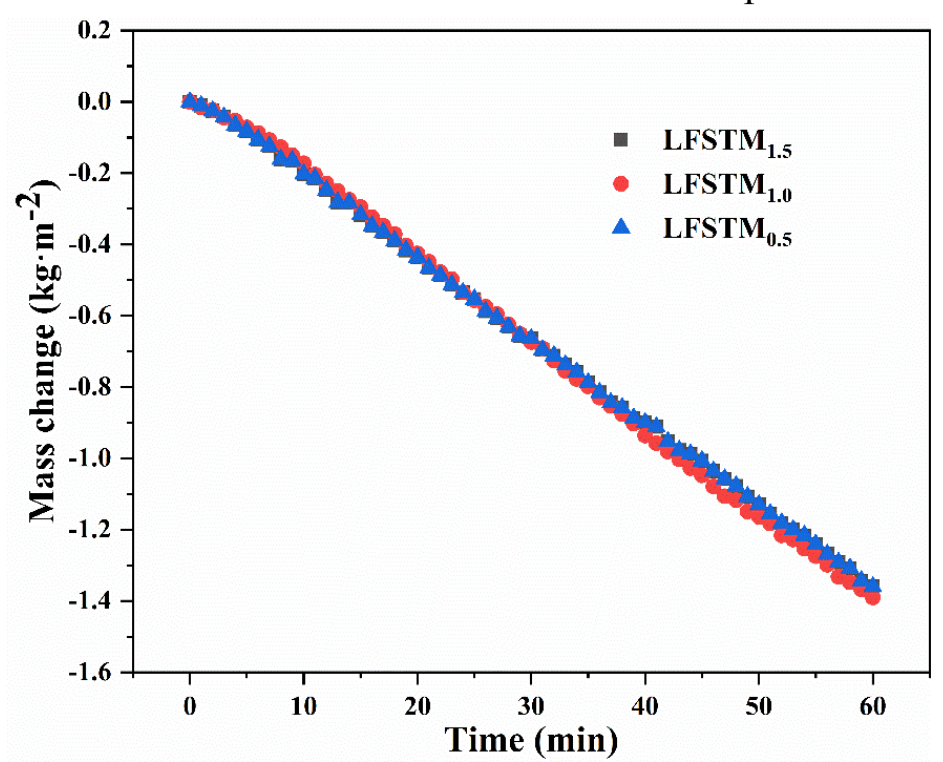

Figure S9 Time-dependent mass change for 20\% saline water by using $\operatorname{LFSTM}_{1.5}$,

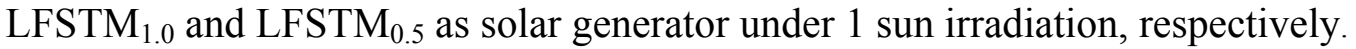




\section{Optimization the number of holes:}

The number of holes is of critical importance for the solar desalination performance of our solar evaporator, as it will influence both the evaporation rate and salt-rejecting behavior of the evaporator. Although the more holes can provide the better salt-rejecting ability, the more holes also lead to increased thermal loss, which will decrease the final evaporation rate. As a result, there is a tradeoff between the efficiency and salt-rejecting properties of the device. Herein, we prepared a series of evaporators with different number of holes and tested the evaporation performance of them in a $20 \mathrm{wt} \% \mathrm{NaCl}$ solution under 1 sun irradiation to find the best number of holes in them. Note that the diameter of hole is $2 \mathrm{~mm}$ and the height is $1 \mathrm{~cm}$. The digital photographs of the devices with different number of holes and the desalination performance of these evaporators are presented in Table S1. After 3 hours of irradiation, significant precipitated salts were observed on the surface of the evaporator with a hole, especially the area marked by yellow lines around the evaporator. Similarly, the surface of the evaporator with three holes also showed precipitated salt in the corresponding yellow lines marked area. The location of the salt deposits is closely related to the location of the holes and the lack of holes leads to insufficient water supply and salt deposition, which will obtain a lower evaporation rate. Interestingly, when the number of holes in the evaporator is increased to 5, there were no precipitated salts. Although there were no salts when the number of holes in the evaporator is 9 , the increase in the number of holes further increases the heat loss, resulting in a lower evaporation rate. Based on these results, The best diameter and the number of holes of the thermal management device was set to $2.0 \mathrm{~mm}$ and 5, respectively.

Table S1 The influence of the number of holes on the salt-rejecting behavior and evaporation rate.

\begin{tabular}{|c|c|c|c|c|}
\hline Holes pattern & $\begin{array}{c}\text { Area ratio } \\
(\%)\end{array}$ & Before & After(3 h later) & $\underset{\substack{\text { Evaporation rate } \\
\left(\mathbf{k g} \cdot \mathbf{m}^{-2} \cdot \mathbf{h}^{-1}\right)}}{\text {. }}$ \\
\hline - & $0.78 \%$ & & & 1.25 \\
\hline & $2.35 \%$ & & & 1.32 \\
\hline$a^{2}$ & $3.92 \%$ & & & 1.45 \\
\hline 1.6 & & & & \\
\hline 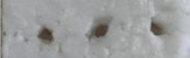 & $7.06 \%$ & & & 1.35 \\
\hline-1 & & & & \\
\hline
\end{tabular}




\section{Optimization the pore size of the weaved fiber:}

As for a solar steam generator, the light absorption and the water supply are two critical factors to determine its solar steam generation performance. The pore size of the weaved fiber will influence both the evaporation rate and salt-rejecting behavior of the evaporator. Weaving holes will affect the evaporation and salt-rejecting performance through the water supply and light absorption area. If the pore size of the weaving is too small, it will block the water supply channel and hinder salt from being discharged in time into the bulk water, thus leading to the precipitation of the salt on the surface of the LFSTM and reducing the evaporation rate of the LFSTM. A pore size of the weaving is too large will reduce the light absorption area, which directly leads to a reduction in light utilization rate and then reduction in evaporation rate. So, a proper pore is optimal to achieve a good evaporation performance for the LFSTM. Based on this line, Herein, we prepared a series of weaved fiber-based evaporators with different pore size for the solar steam generation experiment in a $20 \mathrm{wt} \% \mathrm{NaCl}$ solution under 1 sun irradiation to demonstrate the impacts of pore size of the LFSTM on its solar steam generation performance. The digital photographs of the different number of holes devices and the desalination performance of these evaporators are presented in Table S2. After 1 hours of irradiation, when no holes between the woven fibers, obvious precipitated salts were observed on the surface of evaporator in yellow lines marked zone. In the contrary, there were no precipitated salts when the pore sizes between the woven fibers were about 1 2 and 3 4 mm. Although there were no salts when the pore size was about $3 \sim 4 \mathrm{~mm}$, the increased pore size between the woven fibers is too large will reduce the light absorption area, which directly leads to a reduction in light utilization rate and then reduction in evaporation rate. Based on these results, we set the pore size of weaved fiber is $1 \sim 2 \mathrm{~mm}$.

Table S2 The influence of the pore size of weaved fiber on the salt-rejecting behavior and evaporation rate.

\begin{tabular}{|c|c|c|c|}
\hline $\begin{array}{c}\text { Pore size of weaved fiber } \\
(\mathbf{m m})\end{array}$ & Before & After(1 h later) & $\begin{array}{c}\text { Evaporation rate } \\
\left(\mathbf{k g} \cdot \mathbf{m}^{-2} \cdot \mathbf{h}^{-1}\right)\end{array}$ \\
\hline$\sim \mathbf{0}$ & & & 1.40 \\
\hline $1 \sim 2$ & & & 1.45 \\
\hline $3 \sim 4$ & & & 1.34 \\
\hline
\end{tabular}




\section{Stability test of the LFSTM}

In our work, the candle soot and linen fabrics was bonded by using the polyurethane (PU) as a binder through a physical crosslink. To demonstrate the stability of the as-prepared solar generator, we then designed the following experiment: the sample was bent 1000 times and sonicated of 3 hours in clean water, respectively. As shown in Fig. S10, after 1000 times bending, there is no obvious drop of candle soot and the color of LFS has no obvious change. As illustrated in Fig. S11, after 3 hours sonication, there no obvious candle soot dropped into the water. Most importantly, the color of LFS sample did not change obviously. At the same time, the evaporation performance for pure water under 1 sun illumination was evaluated by using the samples after bending and ultrasonic tests. As shown in Fig. S12, compared with original LFSTM for pure water, after bending or ultrasonic tests, the evaporation performance of LFSTM are substantially unchanged. As shown in Fig. S13, the evaporation rates and energy conversion efficiencies of LFSTM for original sample, bended sample and ultrasonic sample was calculated to be $1.5488 \mathrm{~kg} \cdot \mathrm{m}^{-2} \cdot \mathrm{h}^{-1}(96.8 \%), 1.5446$ $\mathrm{kg} \cdot \mathrm{m}^{-2} \cdot \mathrm{h}^{-1}(96.6 \%)$ and $1.5366 \mathrm{~kg} \cdot \mathrm{m}^{-2} \cdot \mathrm{h}^{-1}(95.9 \%)$, respectively. Therefore, the candle soot is strongly attached on the fiber and the sample has good stability.

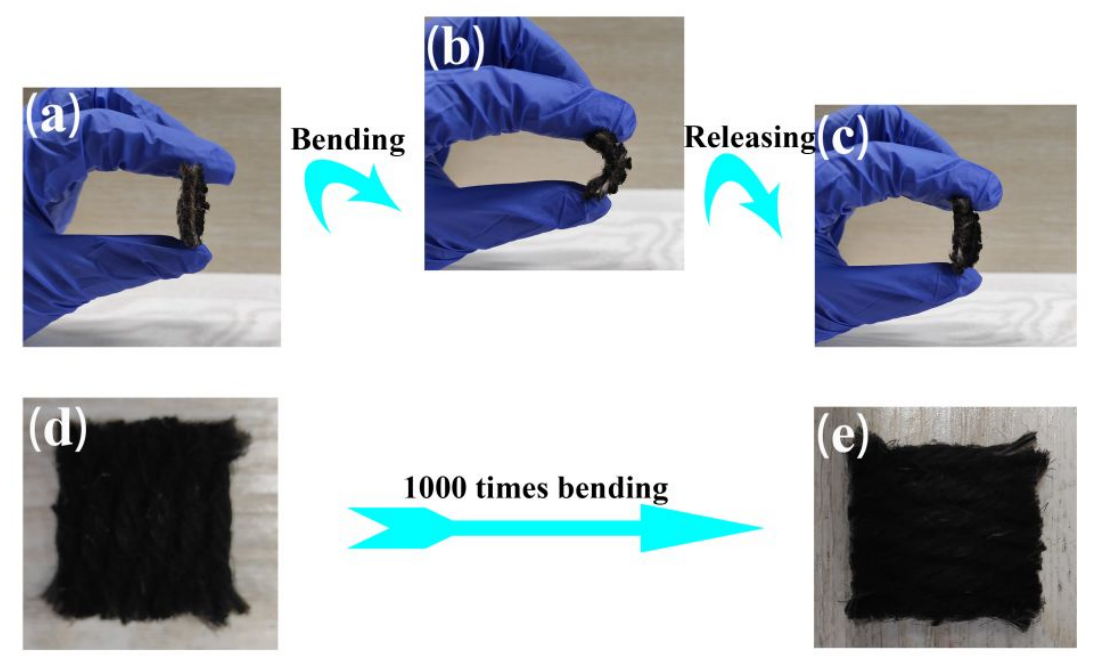

Fig. S10 Digital photographs of the LFS before and after bending, and each time of step (a), (b), (c) is a cycle. (d) and (e) are the digital photographs of LFS before and after 1000 times bending. 


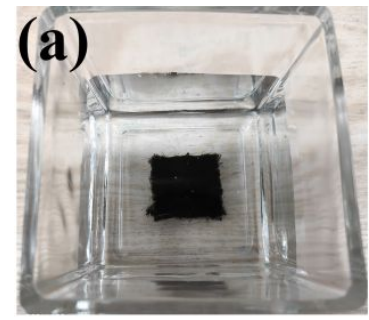

$\mathbf{0 ~ h}$

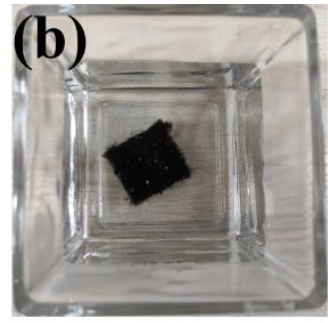

$1 \mathrm{~h}$

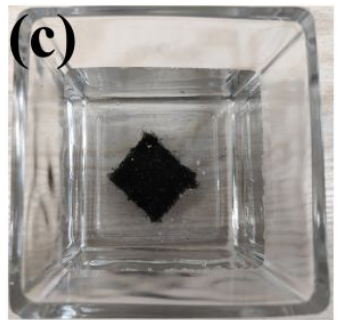

$2 \mathrm{~h}$

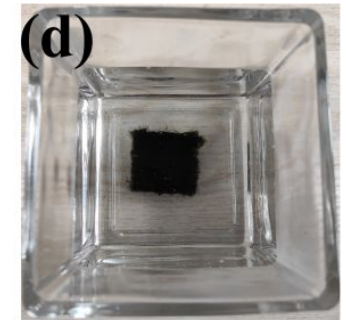

$3 \mathbf{h}$

Fig. S11 Digital photographs of LFS before and after sonication. (a) is the digital photographs of LFS before sonication. (b), (c) and (d) are the digital photographs of LFS after $1 \mathrm{~h}, 2 \mathrm{~h}$ and $3 \mathrm{~h}$ respectively.

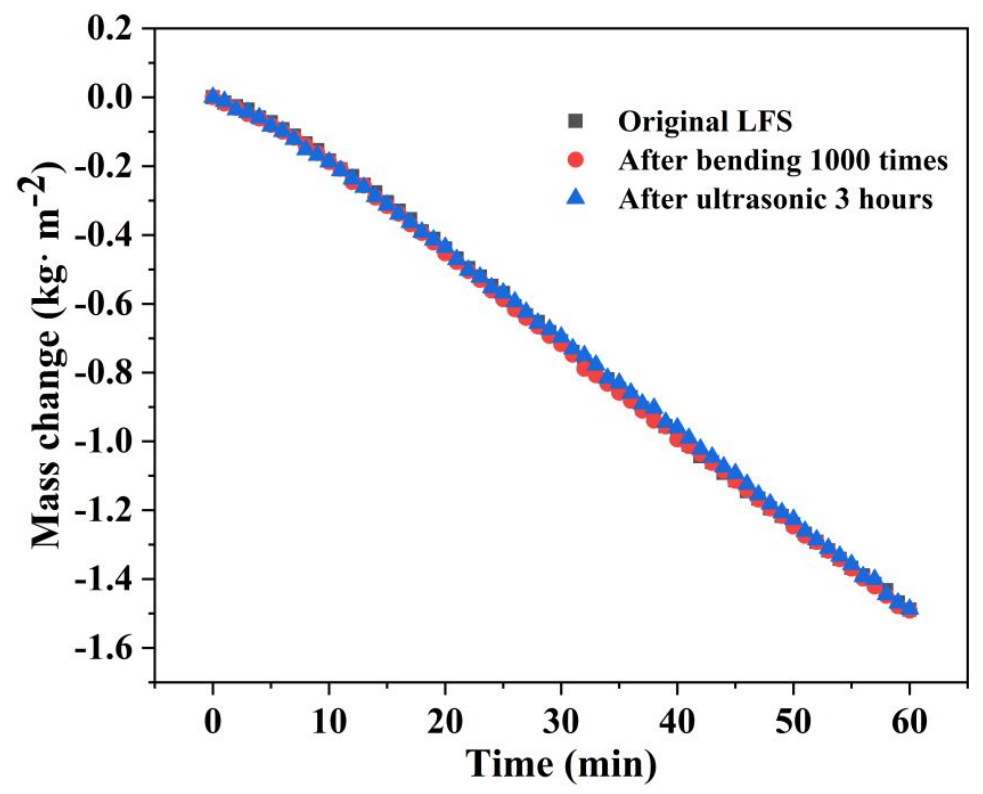

Fig. S12 Time-dependent mass change for LFS of original, after bending 1000 times and after ultrasonic 3 hours under 1 sun irradiation.

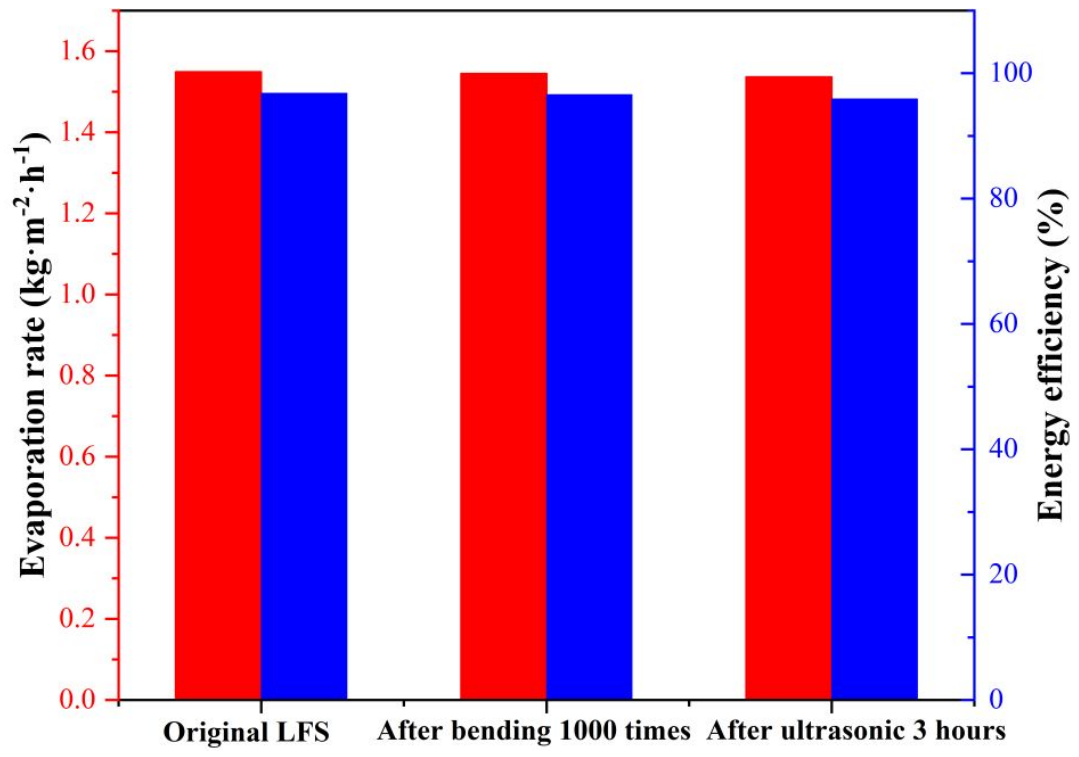

Fig. S13 The evaporation rate and energy efficiency for LFS of original, after bending 1000 times and after ultrasonic 3 hours under 1 sun irradiation. 


\section{Outdoor experiments}

A LFSTM-based solar steam generator with a diameter of $10 \mathrm{~cm}$ was prepared and used as the absorber to carry out the outdoor solar steam generation experiment under the natural solar light. As shown in Figure S14, in the evaporation chamber, simulated seawater in the middle reservoir is evaporated while the purified evaporated water is condensed on the top transparent glass and the purified condensed water droplet moves to the bottom collecting container. This device was operated under the natural solar light for 7 days, excluding the obviously dusky and rainy days. The solar flux and temperature of typical sunny and cloudy from 7:00 17:00 are shown in Fig. S15. As illustrated in Fig. S16, The daily clean water reached $5.4 \sim 6.1 \mathrm{~kg} \mathrm{~m}^{-2}$ on sunny days and $3.0-4.2 \mathrm{~kg} \mathrm{~m}^{-2}$ on cloudy days, which can meet one adult's daily water need $(\sim 2.5 \mathrm{~L})$. It should be noted that the average evaporation rate of outdoors $\left(\sim 0.6 \mathrm{~kg} \cdot \mathrm{h}^{-1} \cdot \mathrm{m}^{-2}\right.$ on sunny) is lower than that in the laboratory $\left(\sim 1.5 \mathrm{~kg} \cdot \mathrm{h}^{-1} \cdot \mathrm{m}^{-2}\right.$ under 1 sun simulated light). We think there are the following reasons. Firstly, it should be clear that the simulated optical power density of the laboratory is $1000 \mathrm{~W} \cdot \mathrm{m}^{-2}$ while the natural solar density only 660 $\mathrm{W} \cdot \mathrm{m}^{-2}$. In addition, due to the movement of the sun's position, the sun not always shines directly on the sample surface, so the natural solar density is much less than $660 \mathrm{~W} \cdot \mathrm{m}^{-2}$. Secondly, the closed environment of the outdoor experiment is different from the open environment of the laboratory. As the evaporation progresses, the humidity in the collection device continues to increase and is not conducive to evaporation, at the same time, condensed water droplets on the surface of the collector will also reduce the illumination of light.( Joule 2019, 3 (3), 683-718.)

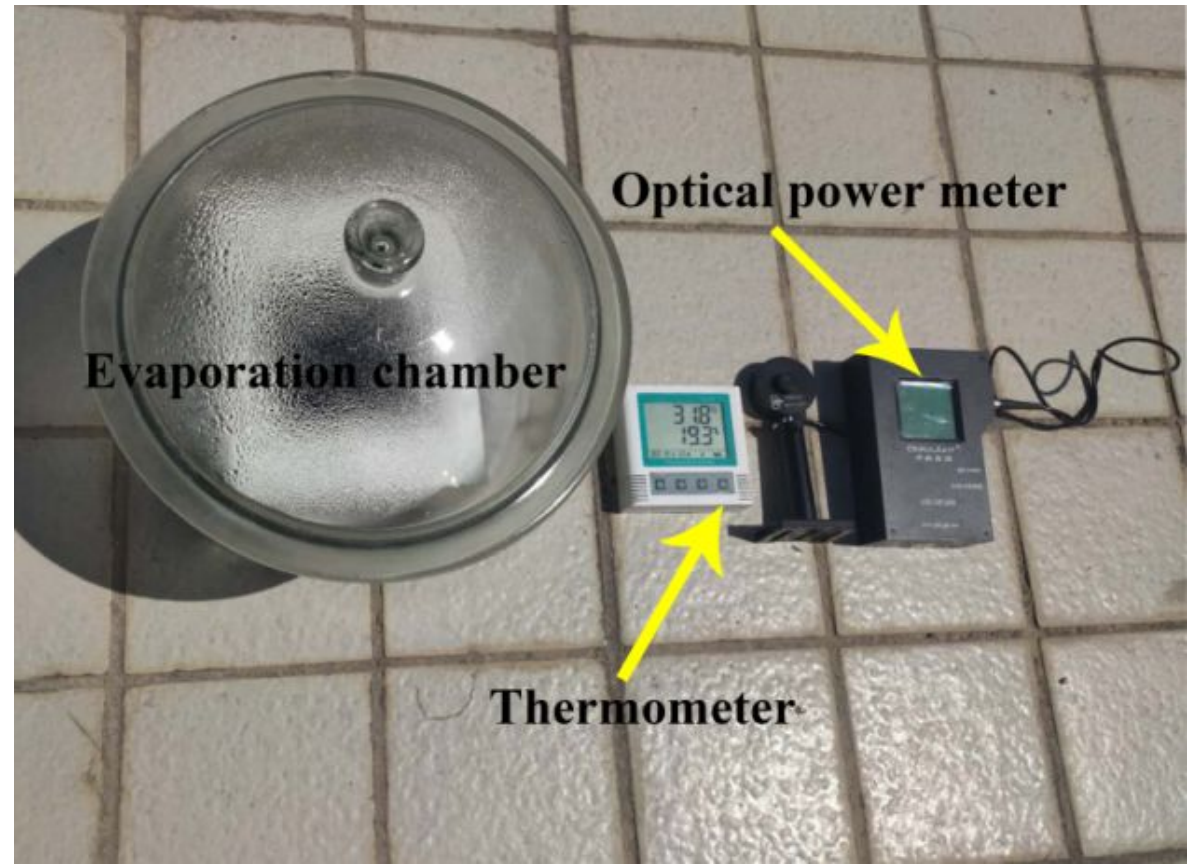

Fig. S14 Digital photographs of the outdoor solar steam generation experiment 

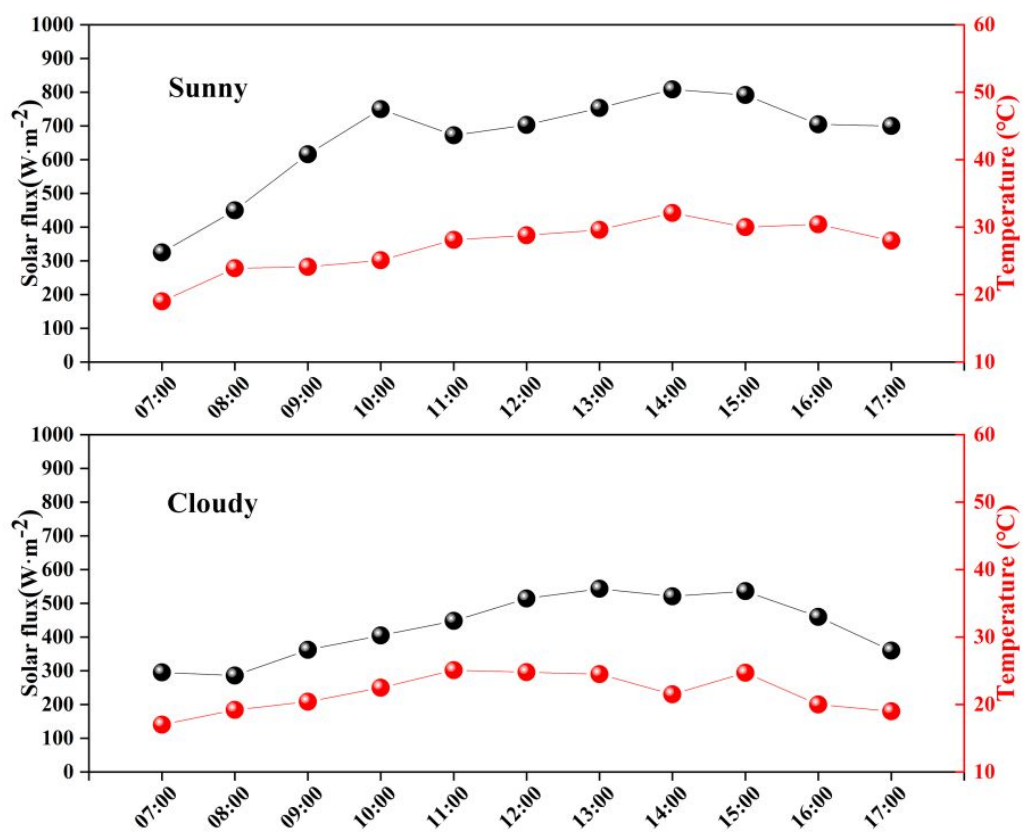

Fig. S15 Typical solar flux and temperature change during 7:00 17:00 on a sunny day and cloudy day

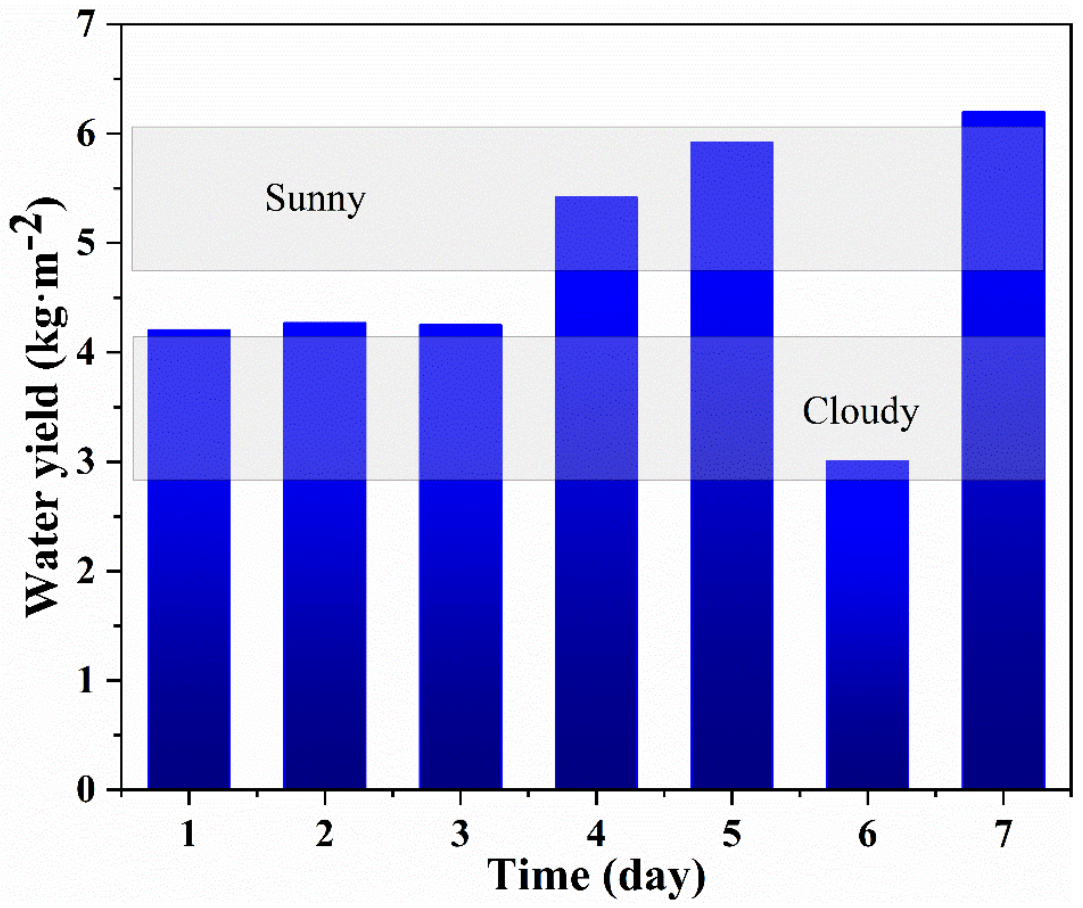

Fig. S16 Collected water in the outdoor installation absorber during a long-term test 
Table S3. The comparison of steam generation performance of the LFSTM and the previously reported at different concentration saline water under 1 sun irradiation.

\begin{tabular}{cccc}
\hline Reference & Salinity ofthe solution & $\begin{array}{c}\text { Evaporation } \\
\text { rate under } \mathbf{1 ~ s u n} \\
\left(\mathrm{kg} \cdot \mathrm{m}^{-2} \cdot \mathrm{h}^{-1}\right)\end{array}$ & $\begin{array}{c}\text { Evaporation } \\
\text { efficiency under 1 } \\
\text { sun }\end{array}$ \\
\hline 1 & $20 \mathrm{wt} \%$ & 1.04 & $75 \%$ \\
2 & $0 \mathrm{wt} \%$ & 1.3 & $725 \%$ \\
3 & $0 \mathrm{wt} \%$ & 1.31 & $79.80 \%$ \\
3 & $10 \mathrm{wt} \%$ & 1.28 & $78.50 \%$ \\
4 & $10 \mathrm{wt} \%$ & 1.2 & $/$ \\
4 & $15 \mathrm{wt} \%$ & 1.16 & $/$ \\
4 & $20 \mathrm{wt} \%$ & 1.12 & $/$ \\
5 & $0 \mathrm{wt} \%$ & 1.24 & $83.10 \%$ \\
6 & $3.5 \mathrm{wt} \%$ & 1.42 & $/$ \\
7 & $3.5 \mathrm{wt} \%$ & 1.42 & $81.20 \%$ \\
8 & $15 \mathrm{wt} \%$ & 0.8 & $57 \%$ \\
9 & $0 \mathrm{wt} \%$ & 1.59 & $93.40 \%$ \\
10 & $0 \mathrm{wt} \%$ & 1.39 & $90 \%$ \\
& $0 \mathrm{wt} \%$ & 1.545 & $96.53 \%$ \\
This work & $3.5 \mathrm{wt} \%$ & 1.498 & $93.38 \%$ \\
& $5 \mathrm{wt} \%$ & 1.482 & $92.32 \%$ \\
& $10 \mathrm{wt} \%$ & 1.467 & $91.35 \%$ \\
& $15 \mathrm{wt} \%$ & 1.448 & $90.07 \%$ \\
\hline
\end{tabular}

Table S4. The total material cost of linen fabric covered candle soot with thermal management. From this estimate, the material cost of the LFSTM is around \$5.62 per square meter of solar steam generator, which extremely low cost is conducive to expanding production

\begin{tabular}{|c|c|}
\hline Materials & Price \\
\hline Linen fiber & $\$ 5.02 / \mathrm{m}^{2}$ \\
\hline Candle soot & $\$ 0.03 / \mathrm{m}^{2}$ \\
\hline Polystyrene foam $(2.5 \mathrm{~cm}$ thick $)$ & $\$ 0.57 / \mathrm{m}^{2}$ \\
\hline Total & $\$ 5.62 / \mathrm{m}^{2}$ \\
\hline
\end{tabular}

\section{References}

1. Kuang, Y.; Chen, C.; He, S.; Hitz, E. M.; Wang, Y.; Gan, W.; Mi, R.; Hu, L., A High-Performance Self-Regenerating Solar Evaporator for Continuous Water Desalination. Adv. Mater. 2019, 31, 1900498-1900505.

2. Xu, W.; Hu, X.; Zhuang, S.; Wang, Y.; Li, X.; Zhou, L.; Zhu, S.; Zhu, J., Flexible and Salt Resistant Janus Absorbers by Electrospinning for Stable and Efficient Solar Desalination. Adv. Energy Mater. 
2018, $8,1702884-1702890$.

3. Xu, N.; Li, J.; Wang, Y.; Fang, C.; Li, X.; Wang, Y.; Zhou, L.; Zhu, B.; Wu, Z.; Zhu, S.; Zhu, J., A Water Lily-Inspired Hierarchical Design for Stable and Efficient Solar Evaporation of High-Salinity Brine. Sci. Adv. 2019, 5, eaaw7013-eaaw7019.

4. Shi, Y.; Zhang, C.; Li, R.; Zhuo, S.; Jin, Y.; Shi, L.; Hong, S.; Chang, J.; Ong, C.; Wang, P., Solar Evaporator with Controlled Salt Precipitation for Zero Liquid Discharge Desalination. Environ. Sci. Technol. 2018, 52, 11822-11830.

5. Jin, Y.; Chang, J.; Shi, Y.; Shi, L.; Hong, S.; Wang, P., A Highly Flexible and Washable Nonwoven Photothermal Cloth for Efficient and Practical Solar Steam Generation. J. Mater. Chem. A 2018, 6, 7942-7949.

6. Xu, Y.; Liu, D.; Xiang, H.; Ren, S.; Zhu, Z.; Liu, D.; Xu, H.; Cui, F.; Wang, W., Easily Scaled-Up Photo-Thermal Membrane with Structure-Dependent Auto-Cleaning Feature for High-Efficient Solar Desalination. J. Membr. Sci. 2019, 586, 222-230.

7. Xia, Y.; Hou, Q.; Jubaer, H.; Li, Y.; Kang, Y.; Yuan, S.; Liu, H.; Woo, M. W.; Zhang, L.; Gao, L.; Wang, H.; Zhang, X., Spatially Isolating Salt Crystallisation from Water Evaporation for Continuous Solar Steam Generation and Salt Harvesting. Energy Environ. Sci. 2019, 12, 1840-1847.

8. He, S.; Chen, C.; Kuang, Y.; Mi, R.; Liu, Y.; Pei, Y.; Kong, W.; Gan, W.; Xie, H.; Hitz, E.; Jia, C.; Chen, X.; Gong, A.; Liao, J.; Li, J.; Ren, Z. J.; Yang, B.; Das, S.; Hu, L., Nature-Inspired Salt Resistant Bimodal Porous Solar Evaporator for Efficient and Stable Water Desalination. Energy Environ. Sci. 2019, 12, 1558-1567.

9. Mu, P.; Zhang, Z.; Bai, W.; He, J.; Sun, H.; Zhu, Z.; Liang, W.; Li, A., Superwetting Monolithic Hollow-Carbon-Nanotubes Aerogels with Hierarchically Nanoporous Structure for Efficient Solar Steam Generation. Adv. Energy Mater. 2018, 9, 1802158-1802166. 